\title{
The Value of Exercise Stress Testing for Renal-Cardiac Risk Assessment in Military Personnel: Exploring the Hidden Half!
}

\author{
Ali Reza Khoshdel ${ }^{1, *}$, Habib Mirzababaie $^{2}$, Hooman Angoorani ${ }^{3}$ \\ ${ }^{1}$ Epidemiology Research Centre, Aja University of Medical Sciences, Tehran, IR Iran \\ ${ }_{3}^{2}$ Air Force General Hospital, Aja University of Medical Sciences, Tehran, IR Iran \\ 3 Department of Sports Medicine, Hazrat Rasool-e-Akram Hospital, Iran University of Medical Sciences, Tehran, IR Iran \\ *Corresponding author: Ali Reza Khoshdel, P.O.Box:16315-781, Tehran, IR Iran. Tel:+98-9121094158, Fax: +98-2188337909, E-mail: a.khoshdel@Ajaums.ac.ir.
}

Received: January 15, 2014; Accepted: January 21, 2014

\begin{abstract}
For decades, the exercise stress test (EST) has been known as a well-established non-invasive diagnostic test in patients with suspected or known cardiovascular disease (CVD). However, EST is also used for several other purposes in the armed forces including fitness assessment and adjustment training in simulated non-conventional environments such as altitude chambers. While exercise stress test is a part of periodic health check-up in specific groups of the military forces such as aviators, divers, and commanders, its diagnostic value for cardiovascular disease (CVD) detection is unclear in asymptomatic healthy subjects with low and intermediate cardiovascular risk according to the current clinical practice guidelines. Furthermore, it is demonstrated that it has a modest sensitivity and specificity for the diagnosis of coronary artery disease (67-72\% and 69-90\% respectively) in symptomatic patients. Hence, the hidden half of the test including non-electrical variations such as hemodynamic changes would be taken into the account or results of other tests such as arterial evaluations would be combined to provide a diagnostic battery. Meanwhile, its performance is also related to the CVD pre-test probability and the prevalence of the disease in the target population. On the other hand, not only the heart, but also vessels and kidneys can be evaluated by EST. Current evidence demonstrates that the hemodynamic response to exercise and exercise-induced micro-albuminuria are associated with cardiac, renal, and arterial functional status. In addition, impaired hemodynamic response to exercise predicts the development of renal dysfunction in the future.

In conclusion, EST would be particularly important in the early detection of cardiac, vascular, and renal diseases in military personnel; however, justification and arrangements for the testing must be individually assessed, and this process can be facilitated by decision analysis methods.
\end{abstract}

Keywords:Exercise Test; Risk Assessment; Diabetes Mellitus

\section{Introduction}

The exercise stress test (EST) is a well-established noninvasive diagnostic test that has been frequently used in patients with suspected or known cardiovascular disease (CVD) for decades (1). Additional applications include the assessment of physical fitness, functional capacity, ventilatory function, metabolic assessment, and the evaluation of peripheral vascular disease (2).

In fact, CVD is a leading cause of mortality in both developed and developing countries. Despite the initial health screening during military forces recruitment, CVD is still considered as an important cause of morbidity, work loss, and mortality among in-duty military personnel and veterans. Therefore, early detection and treatment of CVD is an important goal in military health services.

In addition, CVD is the leading cause of mortality among patients with diabetes mellitus (DM) $(3,4)$, in whom the prevalence, incidence, and mortality from all forms of
CVD is two- to eight-fold higher in comparison to those without DM. Similarly, CVD is the main cause of mortality in renal failure. Therefore, given the increasing epidemic of diabetes in the armed forces, a special attention should be paid to the detection and management of the DM complication including renal and cardiac complications. Subsequent sections of this review will focus on techniques, interpretation, and clinical applications of the EST with particular emphasis on risk stratification for military personnel.

\section{An Overview of Exercise Test}

\subsection{Exercise Equipment}

Cycle ergometers are generally less expensive, smaller, and less noisy than treadmills and produce less upper body motion; however, muscle fatigue in patients who are inexperienced cyclists is a major limitation to reach strategies for military personnel, in particular for officers with critical duties. 
Khoshdel AR et al.

their maximum oxygen uptake $(5,6)$. In contrast, because of the gravitational effects of being upright, walking requires a higher level of myocardial oxygen demand (6) and as a result, a treadmill is more commonly used. Patients who cannot perform leg exercise can be evaluated by arm and upper trunk rowing or cranking. However, because these maneuvers often do not produce adequate workload for cardiac assessment, a pharmacologic stress test is preferred (6).

Since cardiovascular (CV) load can vary considerably among individuals at the same exercise setting (2), energy expenditure should be reported in "estimated metabolic equivalent" (metabolic equivalent of task or METs) of exercise and the nature of the protocol must be clearly specified (1). In the current format, MET refers to a unit of oxygen uptake in a sitting, resting person; one MET is equivalent to $3.5 \mathrm{~mL} \mathrm{O}_{2} / \mathrm{kg} / \mathrm{min}$ oxygen consumption and work activities can be calculated in multiples of METs. One formula for estimating $\mathrm{VO}_{2}$ from treadmill speed and grade is as follows (7):

$\mathrm{VO} 2\left(\mathrm{mLO}_{2} / \mathrm{kg} / \mathrm{min}=(\mathrm{mph} \times 2.68)+(1.8 \times 26.82 \times \mathrm{mph} \times\right.$ grade/100) +3.5

In most patients with CAD, 8 METs workloads are sufficient for angina evaluation. Healthy, sedentary individuals seldom exercise beyond a 10 to 11 METs workload, although physically active individuals including athletes may be able to achieve 16 or more. For functional class I: 7-8 METs; class II: 6 METs and class III: 3-4 METs is tolerable (5). The workload is expressed by watt or kilopond-meters per minute $(\mathrm{kpm})$ when a bicycle ergometer is used. One watt is equivalent to approximately $6 \mathrm{kpm}$ (7). Estimated oxygen costs for cycle ergometer and selected treadmill protocols are presented in Figure 1.

\subsection{Clinical Applications}

The exercise test may be used for diagnostic, prognostic, and therapeutic purposes as well as for physical activity counseling and to design an exercise regimen. However, the recommendations for performing the EST as part of the pre-participation health evaluation in order to initiate or maintain an exercise program, especially a vigorous intensity training program, may differ from those regarding the use of EST to gain information for clinical decision making and management that may include an exercise program. The simple stress test is sometimes combined with other diagnostic means including pharmacologic stress tests, stress echocardiography, radionuclide stress cardiac imaging, and stress testing for artificial pacing.

The most common indication for EST is CAD diagnosis; assessment of cardiac (particularly exercise-induced) arrhythmia, detection of labile hypertension, assessment of functional capacity, evaluation of anti-angina drugs, patient evaluation after bypass surgery or angioplasty, rehabilitation purposes, and research or screening are other indications. The test is contraindicated in acute myocardial infarction (within two days), high risk unstable angina, hemodynamically compromising uncontrolled arrhythmias, symptomatic severe aortic stenosis, uncompensated heart failure, acute pulmonary embolism or infarction, acute endocarditis, myocarditis or pericarditis, hypertrophic cardiomyopathy, acute thrombophlebitis or deep vein thrombosis, and acute aortic dissection. Additionally the test must be performed with precaution in less severe cardiac diseases, high degree conduction defects, severe hypertension (SBP $>200$, DBP $>110)(1,2,5,8)$, and possibly hypotension $(\mathrm{SBP}<90)(9)$.

Figure 1. Estimated Oxygen Cost of Bicycle Ergometer and Selected Treadmill Protocols.

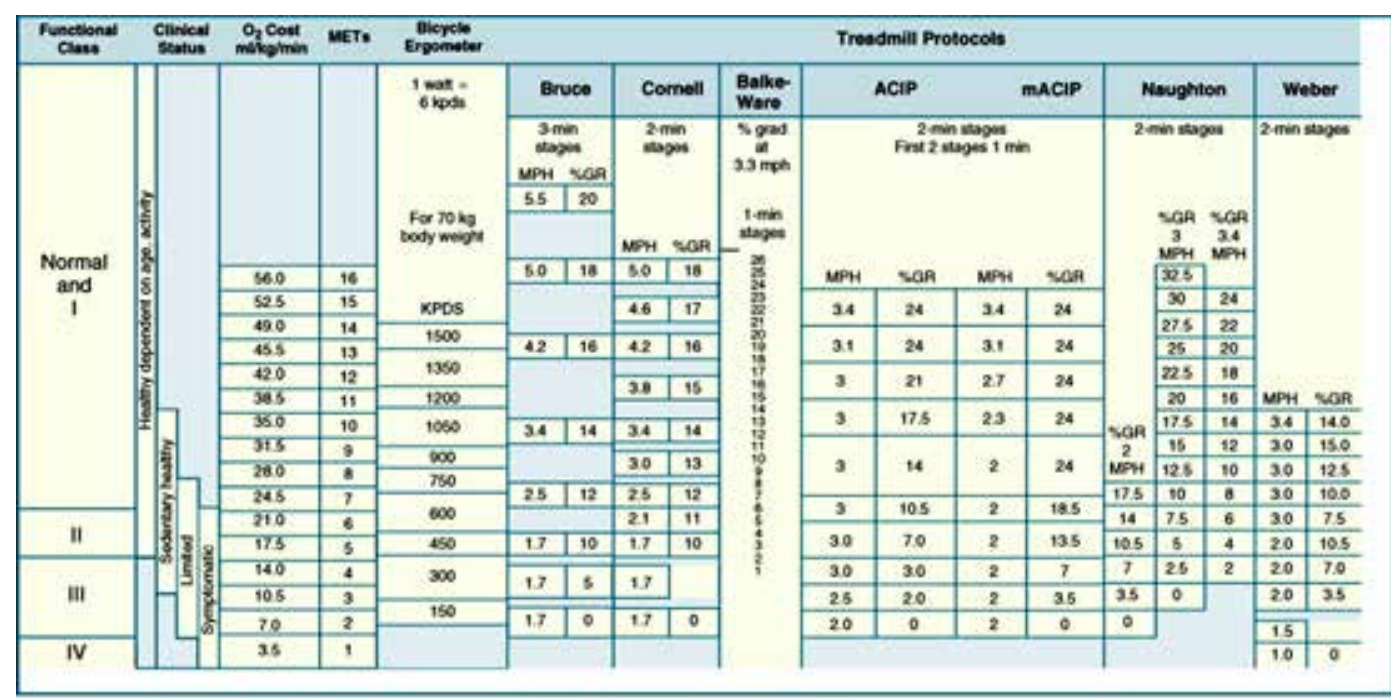

The standard Bruce protocol starts at $1.7 \mathrm{mph}$ and 10 percent grade (5 METs), with larger increments between stages compared to other protocols such as the Naughton, ACIP, and Weber, which start at less than 2 METs at $2 \mathrm{mph}$ and increase by 1-to 1.5-MET increments between stages. The Bruce protocol can be modified by two 3-minute warm-up stages at $1.7 \mathrm{mph}$ and zero percent grade and $1.7 \mathrm{mph}$ and five percent grade. METs $=$ metabolic equivalents. 
Khoshdel AR et al.

\section{Diagnostic Value of Exercise Test}

Physical exercise leads to increased myocardial oxygen consumption via an increased heart rate (HR), intra-myocardial tension, and the velocity of myocardial contraction. The HR acceleration is associated with a reasonably linear increment of myocardial oxygen consumption. In patients with coronary artery disease, coronary blood flow fails to increase adequately to meet myocardium oxygen demands, leading to myocardial ischemia. Myocardial ischemia may manifest by angina, ST segment or $\mathrm{T}$ wave changes, ventricular dysfunction, arrhythmias, or a combinations of the above. There are three major responses to an exercise test in myocardial ischemia: electric events, hemodynamic events, and symptomatic manifestations (Table 1), of which, electric events are the most reliable diagnostic criteria.

\subsection{Electric Events}

One mm or more horizontal or down-sloping ST depression is generally accepted as a definite positive exercise ECG test. In addition, an up-sloping ST depression of $2 \mathrm{~mm}$ or more beyond 0.08 second in duration from J point suggests a positive result $(5,6,8)$. ST elevation is uncommon, and it is almost always encountered in patients with previous MI associated with ventricular aneurysm or ventricular wall dyskinesia; hence, its presence with a previous Q-wave is not considered as positive result (8). P-wave morphology has also been used for CAD diagnosis (10).

\subsection{Hemodynamic Criteria}

A relatively recent development in our understanding of exercise testing is from the data regarding the prognostic significance of hemodynamic responses during and immediately after exercise testing $(11,12)$. Lauer has presented evidences showing that patients who cannot achieve an adequate $\mathrm{HR}$ response to exercise (i.e. chronotropic incompetence) have an unfavorable prognosis beyond that accounted for by only symptoms or ECG changes. The most widely accepted cut point is a failure to achieve $80 \%$ of the age-predicted maximal HR in patients with no pharmacologic reason to have a limitation in their HR response. The prognostic value of a poor HR response is as significant as an exercise-induced myocardial perfusion deficit. An abnormal chronotropic response apparently provides information that is independent of myocardial perfusion because the combination of the perfusion deficit and an abnormal chronotropic index suggests a worse prognosis than either abnormality alone. In a similar fashion, the failure of the HR to recover promptly after exercise provides independent information related to prognosis. A failure of the HR to decrease by at least 12 beats per minute (bpm) by the end of the first minute or $22 \mathrm{bpm}$ by the end of the second minute of recovery are strongly associated with future mortality (13).

The development of exercise-induced hypotension with typical angina pectoris is a strong evidence for CAD. Failure to increase SBP by 10 to $30 \mathrm{mmHg}$ during exercise testing is shown to be an independent predictor of adverse outcome, particularly in post myocardial infarction tests $(5,14)$. On the other hand, more than the expected rise in SBP during exercise most likely represents labile hypertension. Insignificant sinus rate acceleration (less than 120 beats per minutes) during sub-maximal or maximal exercise may be indicative of sick sinus syndrome. It is noteworthy that very anxious subjects with excessive sympathetic tone at the beginning of the exercise may show a temporary reduction of their HR and SBP in the first 2-3 minutes.

An absolute peak systolic pressure of $250 \mathrm{mmHg}$ or a relative increase of $140 \mathrm{mmHg}$ above resting levels is considered a hypertensive response and is predictive of future resting hypertension. A decrease in systolic BP below the resting value, or by $10 \mathrm{mmHg}$ after a preliminary increase, particularly in the presence of other indices of ischemia, is generally abnormal. A systolic blood pressure of $250 \mathrm{mmHg}$ is a relative indication to abort a test. There is typically no change or a slight decrease in diastolic BP during an exercise test. An increase of $10 \mathrm{mmHg}$ in diastolic BP is generally considered as an abnormal finding and may occur with exertional ischemia; the same is true about an increase in dialectic BP to $115 \mathrm{mmHg}(15)$.

Table 1. Available Measurements From Exercise Treadmill Test

\begin{tabular}{lll}
\hline Electrocardiographic & Hemodynamic & Symptomatic \\
\hline Maximum ST depression & Maximum exercise HR & Exercise-induced angina \\
Maximum ST elevation & Maximum exercise SBP & Exercise limiting symptoms \\
ST change slop(up or down sloping or horizontal) & Maximum exercise double product(SBP.HR) & Time to onset of angina \\
Number of leads showing ST changes & Total exercise duration & \\
Duration of ST deviation to recovery & Exertional hypotension & \\
ST/HR indexes & Chronotropic incompetence & \\
\hline
\end{tabular}

Exercise-induced ventricular arrhythmia

Time of onset of ST deviation 
Normally, during the post-exercise period, systolic BP decreases promptly. Several recent investigators have demonstrated that a delay in the recovery of systolic BP is highly related to both ischemic abnormalities and a poor prognosis. As a general principle, the three minutes post-exercise systolic BP should be $90 \%$ of the systolic BP at the peak exercise. If peak exercise BP is not available, the three minutes post-exercise systolic BP should be less than the systolic BP measured one min after exercise $(16,17)$.

\subsection{Symptoms Indicating $C A D$}

Functional capacity can be reported by the tolerated exercise stage and achieved METs. Exercise-limiting symptoms include angina, dyspnea, fatigue, muscular pain, palpitation, dizziness, and claudication. Both the presence and the timing of angina are important in CAD diagnosis. Among other commonly cited symptoms, dyspnea is of particular interest, since it may be a sign of occult left ventricular dysfunction, non-cardiac disease (especially pulmonary disorders such as chronic bronchitis or emphysema), or possibly, an exertional "angina equivalent" (18).

\section{Hemodynamic Response to Exercise}

\subsection{Normal Response}

The normal hemodynamic response to exercise includes tachycardia and enhanced cardiac contractility due to increased sympathetic discharge and simultaneous parasympathetic inhibition. Blood flow redistribution, splanchnic vasoconstriction, and local vasoactive metabolites lead to diversion of blood to the myocardium and active muscles shortly after initiating exercise. In addition, arterial pressure increases roughly parallel to the severity of exercise, mainly due to the enhanced cardiac output. Systolic pressure usually increases more than diastolic pressure, which results in an increased pulse pressure (PP) (19). Although stroke volume usually increases with exercise, the primary cause of increased cardiac output is an augmented HR, which in turn, is due to the vagal withdrawal followed by the sympathetic outflow (8).

\subsection{Abnormal Response}

Aging and hypertension change the exercise response. Cardiac output during exercise is reduced in older and hypertensive patients due to decreased left ventricular (LV) compliance, diastolic filling, and therefore, decreased stroke volume. In addition, maximal exercise HR is reduced in the elderly, possibly due to decreased beta-adrenergic responsiveness. However, the increase in SBP is usually greater with exercise in the elderly, probably due to reduced aortic elasticity and baroreceptor dysfunctional (20). An impaired HR and cardiac output response to exercise occurs in elderly (but not young) patients with non-DM hypertension, possibly due to the reduced left ventricular compliance and impaired stroke volume. An impaired HR response to exercise in the elderly is associated with reduced beta-adrenergic responsiveness (20). On the other hand, Khan reported that the severity of cardiac autonomic neuropathy inversely correlated with a maximal increase in HR and pressurerate product after exercise (21). Nevertheless, baroreflex abnormalities, which commonly occur with or without overt autonomic neuropathy (22), may be attributed to the impaired $\mathrm{HR}$ response amongst the elderly.

The exercise-induced increase in SBP is greater in the elderly. This is mainly attributed to arterial stiffness and impaired arterial vasodilatory function $(19,20,23,24)$. In addition, Poirier et al. found that maximal treadmill performance was higher in subjects with normal diastolic function compared to those with left ventricular dysfunction (25). Thus, diastolic function may also affect the SBP response to exercise. Furthermore, both symptomatic and subclinical silent myocardial ischemia may also contribute to the blood pressure response to the exercise $(8,26,27)$. Finally, impaired renal function may alter the $\mathrm{BP}$ response to the exercise since an increased incidence of CAD and cardiac mortality $(28,29)$, a reduced vagallymediated tachycardic response (30), and endothelial dysfunction have been observed in this population, even before the development of atherosclerotic lesions (28). This has been observed in the early stages of chronic kidney disease (31) and raises the possibility that changes in the cardiac and renal function may be inter-related and may develop in parallel. However, there is no directly relevant longitudinal study to examine this hypothesis and renal function might simply have a direct effect on left ventricular function and exercise capacity.

Although an impaired exercise capacity in patients with DM in comparison to the age- and activity-matched normal subjects has been reported $(32,33)$, the available evidence on the hemodynamic response to the exercise in DMs is contradictory. A few years ago, we reported an impaired exercise capacity, circulatory power, HR, and systolic blood pressure response to EST in patients with DM even among patients with low CV risk (34) and raised the question of potential impact of pulse pressure, arterial compliance, and diastolic dysfunction in this regard.

Several complex indices have also been used for better describing the hemodynamic response to the exercise, including maximal predicted heart rate (MPHR) and heart rate reserve (HRR) as chronotropic indices as well as circulatory power and rate-pressure product (double product) as representatives of myocardial functional status. These are calculated as follows (35-38):

$\mathrm{MPHR}=\mathbf{2 2 0}$ - age

HRR $=[($ Peak HR - HR arrest $) /(220-$ age - HR arrest $)] \times 100$

Circulation power $=\mathrm{O} 2 \times \max \mathrm{SBP}$

Rate - pressure product $=(\max H R-\max S B P) / 100$ 


\section{Exercise-Induced Albuminuria}

Current evidence shows that albuminuria not only is a marker of renal disease, but also represents CV risk (39). Furthermore, microalbuminuria is an independent predictor of the exercise tolerance (40), left ventricular function (41, 42), and further development of CAD (43). Moreover, asymptomatic patients with DM and microalbuminuria are more likely to have a positive exercise test results $(44,45)$. Therefore, given this increased pre-test probability, the test results are expected to be more accurate in patients with DM and microalbuminuria.

In the first stage of DM, urinary albumin excretion is typically normal but transient microalbuminuria (evoked by stress, physical exercise, poor glycemic control, and intercurrent febrile illness) may occur. Thus, induction of proteinuria by physical stress, sympathetic stimulation, oral or intravenous loading tests (e.g. saline or protein), and the use of medical substances (e.g. dexamethasone) may unmask sub-clinical proteinuria. Exercise-induced proteinuria includes both albumin and IgG in patients with DM but only albumin in healthy individuals (46). However, exercise-induced proteinuria varies in diverse situations. For instance, measurements of four endogenous proteins before, during, and after exercise in patients with DM and normal urine albumin demonstrated that in normal subjects and non-obese patients with DM, exercise-induced microproteinuria is probably of both glomerular and tubular origin, yet in patients with obesity and DM it is mainly glomerular in origin (47). However, regardless of origin, basal and exercise-induced proteinuria have been reported to be significantly higher in patients with DM than in healthy controls (48). Additionally, there are evidences indicating that exercise-induced albuminuria might be an early sign of diabetic nephropathy and predictive of further irreversible changes of diabetic renal disease (49-51). As a result of these studies, both qualitative and quantitative evaluation of exercise-induced proteinuria might be helpful in the early diagnosis of renal damage and provide additional information relating to $\mathrm{CV}$ health. In fact, mi- croalbuminuria reflects a general arterial damage, which may present as a cardiac disease, renal dysfunction, hypertension, and cerebrovascular manifestations. Thus, it has been suggested that a combination of blood pressure profile, microalbuminuria (at rest or after exercise), and arterial assessment may help in risk assessment in very early stages of circulatory derangement (52).

\section{Assessment of Arterial Stiffness}

Arterial stiffness is an early presentation of arteriosclerosis and can be non-invasively estimated in outpatient clinical practice (53). It is an independent predictor of all-cause and CV mortality in various groups of patients (54-56) and is associated with early stages of DM and chronic renal failure (57). Interestingly, our recent studies demonstrated that hemodynamic response to EST is also correlated with the degree of arterial stiffness (58). Moreover, evidence-based approach to the risk stratification of suspicious CVD could be improved by measuring arterial stiffness (52). Thus, implementation of arterial studies in combination with EST may help in a better risk management of the military population.

\section{Epidemiologic Aspects of Cardiac Stress Test}

\subsection{Diagnostic Performance}

A meta-analysis of 147 consecutively published reports (Table 2) involving 24074 patients who underwent both coronary angiography and a stress test revealed a wide variability in its sensitivity and specificity, although exclusion of possible confounders improved the sensitivity and specificity to $67-72 \%$ and $69-90 \%$, respectively (5).

Test sensitivity may be reduced if $85 \%$ of the maximum predicted heart rate is not achieved (8), in the presence of left ventricular hypertrophy, resting ST changes in the ECG, and taking medications including digoxin (5).

Table 2. Meta-analyses of Exercise Test Performance

\begin{tabular}{|c|c|c|c|c|c|}
\hline Grouping & Number of Studies & Total Number & Sensitivity, \% & Specificity, \% & $\begin{array}{l}\text { Predictive Ac- } \\
\text { curacy, \% }\end{array}$ \\
\hline Meta-analysis of standard EST $^{\mathrm{a}}$ & 147 & 24047 & 68 & 77 & 73 \\
\hline Meta-analysis without MI $^{\mathrm{a}}$ & 58 & 11691 & 67 & 72 & 69 \\
\hline Meta-analysis without workup bias & 3 & $>1000$ & 50 & 90 & 69 \\
\hline Meta-analysis with $\mathrm{ST}^{\mathrm{a}}$ depression & 22 & 9153 & 69 & 70 & 69 \\
\hline Meta-analysis without ST depression & 3 & 840 & 67 & 84 & 75 \\
\hline Meta-analysis with digoxin & 15 & 6338 & 68 & 74 & 71 \\
\hline Meta-analysis without digoxin & 9 & 3548 & 72 & 69 & 70 \\
\hline Meta-analysis with $\mathrm{LVH}^{\mathrm{a}}$ & 15 & 8016 & 68 & 69 & 68 \\
\hline Meta-analysis without LVH & 10 & 1977 & 72 & 77 & 74 \\
\hline
\end{tabular}

\footnotetext{
${ }^{\mathrm{a}}$ Abbreviations: EST, Exercise stress test; LVH, Left ventricular hypertrophy; MI, Myocardial infarction; ST, stress test
} 
Despite the marked effect of beta-blockers on maximal exercise HR, when the patients were sub-grouped according to beta-blocker administration, no difference in test performance was found (59).

Both individual pre-test probability of CAD (estimated clinically) and CAD prevalence in the target population can affect its diagnostic value $(5,60)$. The frequency of false-positive or false-negative results is directly influenced by the pre-test probability of CAD (the lower the pre-test probability the higher the possibility of false results). Not surprisingly, the test is most valuable in the intermediate pre-test probability category because it can affect the diagnostic decision to the highest degree. The test predictive value is also affected by CAD prevalence in the population. For instance, considering values of 50\% and $90 \%$ for sensitivity and specificity of the test, respectively, a 10-fold increase in the CAD prevalence increases the positive predictive value from 21 to $83 \%$.

A report from Pakistan army (61) evaluated 291 soldiers with chest pain or left arm pain and normal resting ECG. It concluded that EST had 74\% sensitivity and 78\% specificity, while Thallium stress test performed better with $91 \%$ sensitivity and $71 \%$ specificity in comparison to angiography. However, the report has been criticized for sampling and selection bias, since the Thallium stress test was used for patients with greater probability of coronary disease.

Despite this modest accuracy, combining variables into scores has improved exercise stress test performance. Using post-exercise SBP to maximal SBP ratio in combination with monitoring for ST depression, improves the accuracy of treadmill exercise testing (62); the ratio was greater than 0.87 and had $68 \%$ sensitivity and $82 \%$ specificity for detection of coronary stenosis. However, this result was obtained from a small sample size and further investigation is needed to assess its impact on test performance.

\subsection{Prognostic Value}

Exercise testing provides not only diagnostic information but also, more importantly, prognostic data. The sensitivity of EST for the prediction of coronary heart events in a three- to twelve-year follow-up ranged from 40 to $62 \%$ (63). In a large study of asymptomatic, apparently healthy individuals, the risk of cardiac events was ninetime greater when the result was abnormal (7). While silent ischemia is a frequent finding in young individuals, studies have demonstrated that screening of asymptomatic subjects cannot effectively reduce the risk of major CV events (64-66). Furthermore, there is a considerable false positive rate and the lack of a generally accepted strategy for managing false positive cases is still an obstacle to employ it as a screening tool (5).

Mark and colleagues developed a treadmill score that is shown to be accurate in prediction of prognosis and effective in risk estimation in patients younger than 75 years of age (7):
Treadmill score $=$ excersise time $-(5 \times$ ST deviation $)-(4 \times$ treadmill angina index)

This score was used to develop a nomogram that incorporated pre-test disease probability to predict a five-year survival and the annual mortality rate (Figure 2 ).

The most widely accepted and used prediction method is the Duke treadmill Score or the related Duke treadmill normogram. Both of these tools would be appropriate for patients with known or suspected CAD being considered for coronary angiography without a history of a myocardial infarction or revascularization procedure. The Duke score normogram considers exercise capacity, the magnitude of ST-segment depression, and the presence and severity of angina pectoris. The calculated score is shown to be related to annual and five-year survival rates and allows the categorization of patients into low-, moderate-, and high-risk subgroups. This categorization may guide the caring physician to proceed to conservative or aggressive therapies, depending on the classification category that the patient falls into (67).

Interestingly, patients' symptoms also have prognostic value. A large recent epidemiologic study showed that patients with dyspnea had a substantially higher rate of both cardiac and all-cause mortality than those with other or no symptoms at cardiac exercise testing. In contrast, patients with typical angina did not have a higher rate of death. Interestingly, patients with dyspnea had significantly higher rates of DM and hypertension (18).

An association between exercise capacity and CVD has been reported (40) and HR increase in response to exercise has been demonstrated to be predictive against sudden death (68), mortality $(35,36)$, and CAD (69). Nevertheless, our recent finding of a relatively impaired hemodynamic response to EST in patients with DM (34) as well as greater incidence of severe renal failure in patients with impaired hemodynamic response to exercise (70) are of paramount importance in the risk assessment strategies in populations like military personnel.

\section{Exercise Stress Test in Military Forces}

Military personnel are supposed to be completely healthy, physically fit, and less likely to have CAD when compared to their age-matched counterparts selected from general population. However, they would be exposed to unusual environment (such as extreme coldness or warmth, very high altitudes or hypoxia, stress, and emotional deprivation) and must perform extraordinary duties, which need a completely competent CV system.

Currently, EST applications in military forces includes assessment of body fitness, muscle strength, endurance and combat readiness in conventional and unconventional environments, cardiopulmonary evaluation, training, and annual check-ups in specific occupations. From clinical point of view, it is sometimes recommended for CV diagnosis. However, its diagnostic and 
prognostic performance could be hampered by several factors including clinical symptoms, co-morbidities, risk factors, job stress, and the pre-test probability of the disease.

It is important to consider that EST produces other important information that can be used in cardiac, arterial, and renal risk assessment. This fact is commonly overlooked. Given the increasing frequency of non-communicable diseases among military personnel in recent decade, it would bear clinical advantages for early detection and improvement of preventive policies. In a large study, CDC evaluated 284850 active-duty Air Force personnel in US and determined 5.3\% with hypertension, $4.6 \%$ with dyslipidemia, and $0.3 \%$ with DM (71), which is significantly higher than the previous reports of US armed forces a decade ago (72). Our previous investigations in Iran estimated greater rates for Iranian military personnel: hypertension in $25 \%$ of parachutists and $8.8 \%$ in army, while diabetes was detected in $5.2 \%$ of military parachutists and $1.8 \%$ of army $(73,74)$. Meanwhile, metabolic syndrome is estimated to be present in about $18.4 \%$ in Bangladesh army (75), compared to $8.2 \%$ in Iranian army (73, 76 ) and $13.5 \%$ in military parachutists (74). Furthermore, a study in India has recently demonstrated that about 15\% of asymptomatic active-duty armed force personnel had some degrees of chronic renal dysfunction and/or albuminuria (77). According to the aforementioned health profiles, more effective preventive strategies and early detection measures must be incorporated into the periodic health check-up in armed forces and EST would be an important part of such a program.

\subsection{Exercise Stress Test in Asymptomatic Military Personnel}

The United States Preventive Service Task Force (USPSTF) has recently stated that for low-risk asymptomatic individuals resting or exercise ECG does not over-perform usual risk scores (such as Framingham's score) and does not modify the management strategies (64). Even in intermediate or high risk groups, there is no adequate data regarding effectiveness of resting or exercise ECG screening in reduction of $\mathrm{CV}$ events. Thus, given the potential direct or indirect harms of testing, EST is not generally recommended, neither for diagnosis nor for screening (64). However, American College of Cardiology Foundation recommends that resting ECG is reasonable for CV risk assessment in asymptomatic adults with hypertension or DM. The recommendation also includes considering non-ECG markers in EST such as exercise capacity(64). A recent review noted that combining vascular age into the CV assessment, improves discrimination by Framingham's risk score (78). Consequently, the decision would be influenced by other factors in people in certain occupations such as pilots for whom sudden CV events may endanger others or result in failing in critical missions.

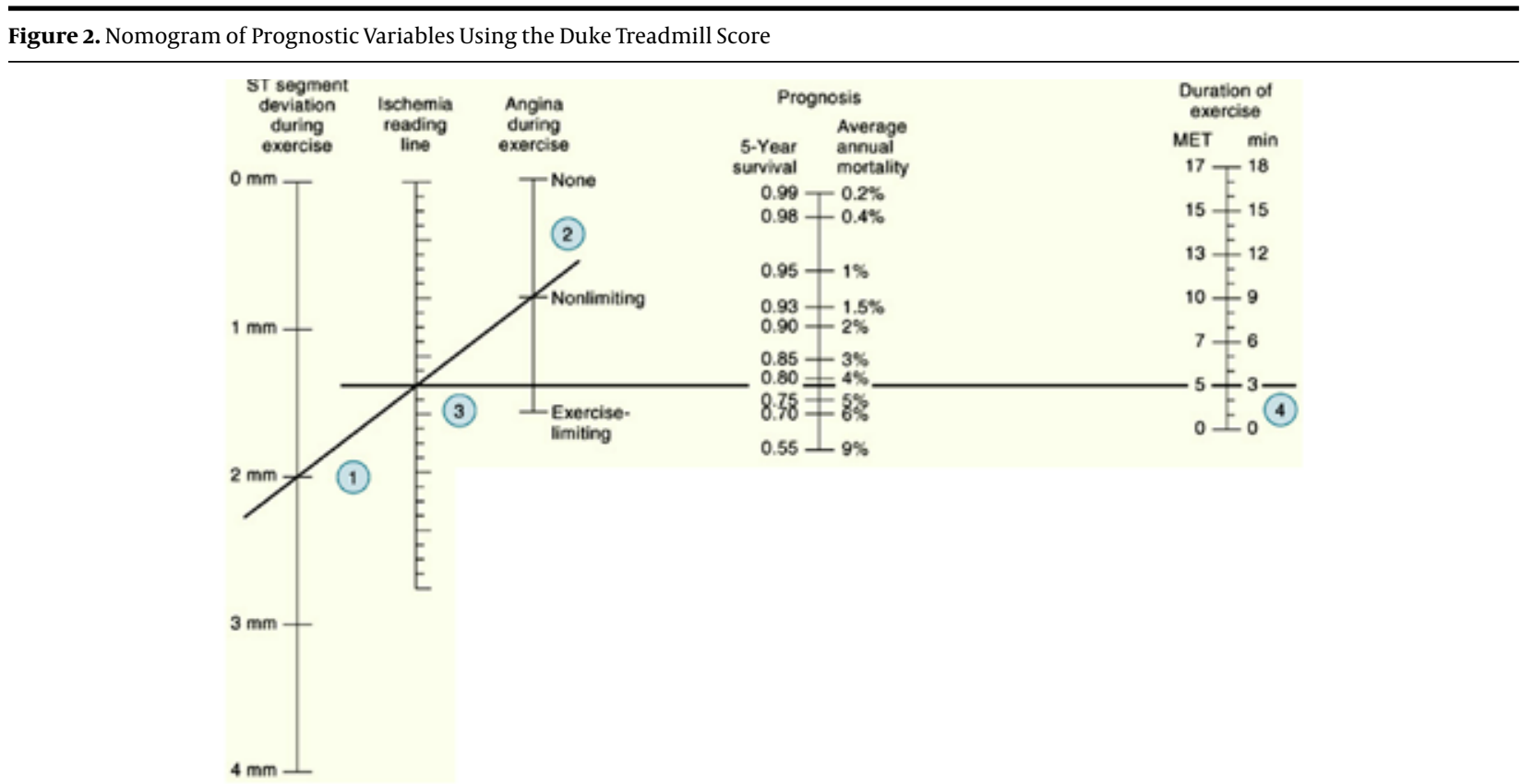

It incorporates duration of exercise (in minutes) - ( $5 \times$ maximal ST segment deviation during or after exercise) (in mm) - $(4 \times$ treadmill angina index) Treadmill angina index is 0 for no angina, 1 for non-limiting angina, and 2 for exercise-limiting angina. The marks on the ischemia reading line and duration of exercise line are connected, and the intersection on the prognosis line determines five-year survival rate and average annual mortality rate for patients with these selected specific variables. MET = Metabolic Equivalent. (Adapted from Mark DE, Shaw L, Harrell FE Jr, et al: Prognostic value of a treadmill exercise score in outpatients with suspected coronary artery disease. N Engl J Med 325:849, 1991. Copyright Massachusetts Medical Society). 


\subsection{Exercise Stress Test in Diabetic Military Personnel}

The special writing group of the American Heart Association established that DM is a "coronary risk equivalent" and indicated that patients with DM belong to the same risk category as patients with known CVD (79). This risk increases with age ( $>35$ years), duration of DM (>10 years), presence of microvascular complications, and other CV risk factors (5). Screening and risk assessment of DM patients is also strongly recommended in many guidelines including a French guideline that recommended screening for silent myocardial ischemia (including EST) in patients with DM and one additional risk factor (79). However, the American Heart Association gave an evidence rating of IIb to EST screening in patients with DM due to limited evidence (5). Nevertheless, they recommended EST in this group when individuals plan to perform moderate to high intensity exercise (5). While the US preventive services task force did not support EST in asymptomatic patients without another CV risk factor, they did not present any recommendation for or against an EST in asymptomatic patients with CV risk factors due to a lack of enough evidence and the potential harms from false positive results (e.g. invasive investigations) (63). In contrast, the consensus statement from the American Diabetes Association and American College of Cardiology suggested non-invasive cardiac testing for patients with DM and one additional risk factor including peripheral arterial disease, cerebrovascular disease, resting ECG abnormalities, or the presence of two or more CVD risk factors. Hence, the decision must be based on the individual clinical judgment (79).

\subsection{Harms of Exercise Stress Testing}

Although EST is generally a safe procedure, there is a small but definite mortality risk of approximately one in 10000 , and two to three per 10,000 tests results in the context of a major morbid event such as myocardial infarction $(1,2,5$, $60)$. Furthermore, direct harms of the tests, adverse events associated with future testing such as angiography or percutaneous coronary angioplasty, and also psychological distresses must be considered(64).

\subsection{Decision Making Analysis}

There is still no consensus regarding exercise stress testing in asymptomatic subjects and currently individual justification is preferred, as opposed to a general recommendation. Meanwhile, a "clinical decision analysis" may help evaluate the utility of such a test for each patient. Decision analysis is a mathematical tool designed to facilitate complex clinical decisions in which many variables must be considered simultaneously. Importantly, individual pre-test probability and variability in the test performance is considered in this method (80).

The analytic procedure is based on the probability and predetermined value (utility) of all possible outcomes of diagnostic or therapeutic options (illustrated on a decision tree) and attempts to simplify the comparison between the available alternatives. A decision tree is a map of all relevant courses of action and their associated outcomes. A branch point representing a diagnostic or therapeutic decision is known as a "decision node" (square node) and the one representing a chance outcome, not directly controlled by the physician, called a "chance node" (circle node).

The probability of outcomes in each branch is determined by available published reports and the probability of the branches related to a diagnostic procedure is estimated from the reported test performance by the "back calculation" method using a "decision matrix" (Table 3). The utility of the outcome is represented on an ordinal scale based on a simple or multidimensional clinical judgment. The expected utility at any given chance node is then estimated by the "folding back" process, i.e. multiplying the probability of each branch by the corresponding utility and then summing the branches emanating from the node. This process provides an expected utility for each possible action and the option with the highest utility is selected. Finally, the cross point between the two actions in a "sensitivity analysis" can determine the decision threshold based on different pre-test probabilities.

Figure 3 is an example of the clinical application of this system when a decision should be made for EST in an asymptomatic 45-year-old patient. He was an Asian smoker colonel in the army with DM (diagnosed five years ago in the annual check-up), total cholesterol of $250 \mathrm{mg} / \mathrm{dL}$, HDL of $20 \mathrm{mg} / \mathrm{dL}$, and systolic blood pressure of $145 \mathrm{mmHg}$ whose $\mathrm{CV}$ risk score is estimated to be $39.2 \%$ risk of CAD in 10 years. The calculations can be changed according to the pre-test probability and the test performance compared with the closest group of subjects to the individual patient. For instance, in an asymptomatic patient with DM, having microalbuminuria may increase the pre-test probability by two to six times (81-83). A similar adjustment could be considered for impaired hemodynamic responses to exercise based on the available evidences (84-86). Importantly, according to our previous findings of significant degrees of arterial stiffness in asymptomatic aviators (87) and based on evidence-based logics (52), the CV risk in our presumptive patients should be increased.

Figure 4 illustrates a decision threshold (above which the test is beneficial) for performing an EST with two values for the test performance (one with a $70 \%$ sensitivity and a $75 \%$ specificity, and another with a $80 \%$ sensitivity and $85 \%$ specificity). The results suggest that the test is beneficial if the pre-test probability of CAD is about 35\% or higher. This is in agreement with the suggestion of Greenland et al. regarding the possible advantages of EST in the intermediate cardiac risk group (88). However, evidences from longitudinal studies are sparse. 
Table 3. An Example of Decision Matrix When Sensitivity Is 70\%, Specificity Is $75 \%$ and the Estimated Pre-test Probability Is $40 \%$.

\begin{tabular}{llll}
\hline & CAD $^{\mathrm{a}}(+)$ & CAD $(-)$ & Total \\
\hline EST $^{\mathrm{a}}+$ & 280 & 150 & 430 \\
EST- & 120 & 450 & 570 \\
Total & 400 & 600 & 1000 \\
\hline
\end{tabular}

a Abbreviations: CAD, coronary artery disease; EST, exercise stress test.

Figure 3. Decision Tree for Exercise Stress Testing in an Asymptomatic Military Officer With Stressful Job

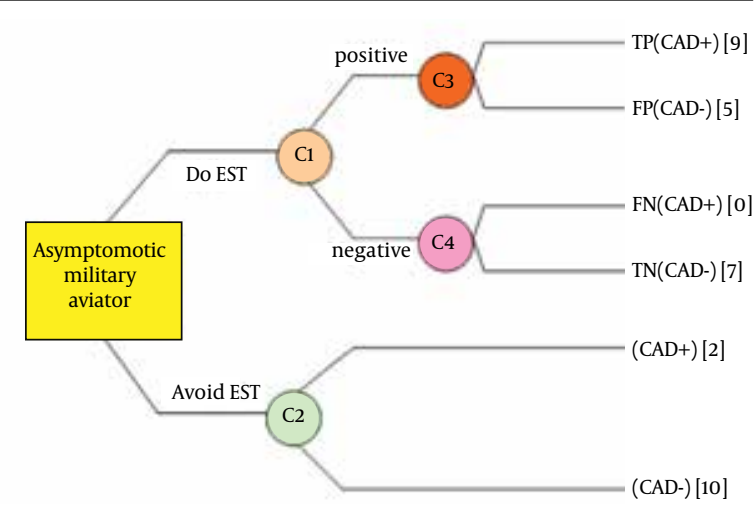

[X] represents utility of the outcome, circles are the chance nodes and the square is the decision node; the calculation must be performed using the disease pre-test probability and the test performance in the target population.

Figure 4. Sensitivity Analysis for Decision About Exercise Stress Test (EST) Given Different Assumptions for Pre-test Probability

\section{A}

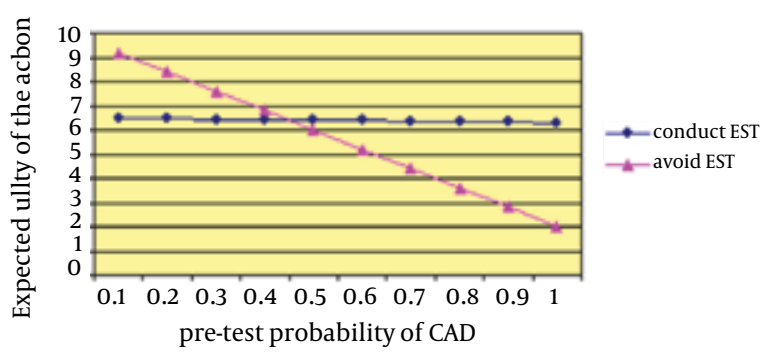

B

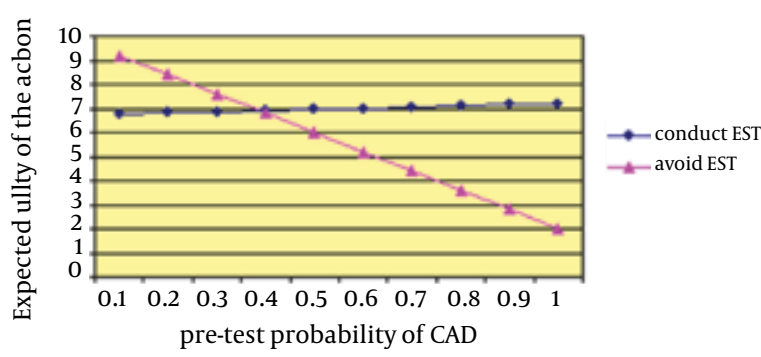

Panel A: Sensitivity $70 \%$ and specificity $75 \%$, Panel b: Sensitivity $80 \%$ and specificity $85 \%$.

\section{Conclusions}

With increasing incidence and prevalence of non-communicable diseases in military populations, it seems that EST would be a useful tool for risk assessment for cardiac, renal, and arterial subclinical pathologies. Although EST yields a significant false positive result rate in low risk groups, it is recommended in military personnel with a medium to high $\mathrm{CV}$ risk status or even in otherwise healthy asymptomatic personnel with DM. It not only assesses cardiopulmonary fitness, but also detects subclinical cardiac ischemia and provides further information about the cardiac conductive system and left ventricular function. Considering electric, hemodynamic, and symptomatic events together increases the diagnostic test performance, and enhances the predictive and prognostic value of the test for detecting further complications of the diseases such as CV events, stroke, kidney damage, and mortality. However, the test performance may vary due to different factors including pre-test probability; consequently, every clinical decision must be made on an individual basis. It can be facilitated by decision analysis methods that are based on individualized criteria.

As experts have suggested concomitant evaluation and treatment of kidney and heart problems (89, 90), a combination of blood pressure profile, arterial stiffness evaluation, exercise-induced albuminuria, and hemodynamic response to exercise may represent the "cardiorenal risk". Hence, the neglected hidden half of the test should be properly explored. EST needs to be better performed, evaluated, reported, and applied in the risk stratification, particularly in high-risk stressful military jobs.

\section{Acknowledgements}

The first author appreciated Professor Saxon White and Professor Shane Carney from the University of Newcastle, Australia, for their precious comments on the value of EST and their collaborations in the previous studies which were mentioned here.

\section{Authors' Contribution}

All authors actively contributed in collection of required information, manuscript writing and reviews.

\section{Financial Disclosure}

There was no financial benefit for the study group, sponsors or the training centers. No conflict of interest was declared.

\section{Funding/Support}

This study was supported by the Epidemiology Research Centre, AJA University of Medical Sciences, Tehran, Iran.

\section{References}

1. Clinical exercise stress testing. Safety and performance guidelines. The Cardiac Society of Australia and New Zealand. Med J Aust.1996;164(5):282-4. 
2. Freedman B. Safety and performance guidelines for clinical exercise stress testing.: On behalf of Rehab Exercise and Prevention Working Group; The cardiac society of Australia and New Zealand (CSANZ); 2003.

3. Benetos A, Adamopoulos C, Bureau JM, Temmar M, Labat C, Bean $\mathrm{K}$, et al. Determinants of accelerated progression of arterial stiffness in normotensive subjects and in treated hypertensive subjects over a 6-year period. Circulation. 2002;105(10):1202-7.

4. Intensive blood-glucose control with sulphonylureas or insulin compared with conventional treatment and risk of complications in patients with type 2 diabetes (UKPDS 33). UK Prospective Diabetes Study (UKPDS) Group. Lancet. 1998;352(9131):837-53.

5. Gibbons RJ, Antman EM. ACC/AHA 2002 Guideline Update For Exercise Testing. ACC/AHA Practice Guidelines. Bethesda, USA: American College of Cardiology; American Heart Association; 2002. Available from: http://my.americanheart.org/idc/groups/ahaeccinternal/.../http://my.americanheart.org/idc/groups/ahaeccinternal.

6. Crawford MH. Diagnostic studies in cardiology. Crawford MH editor. New York: Lange Medical Books, McGraw Hill; 2003.

7. Zipes DP, Libby P, Bonow RO. Braunwald's Heart Disease. 7th ed. Braunwald E editor. Philadelphia: Elsevier Saunders; 2005.

8. Eisenberg JM, Hutton JJ, Klippel JH, Kohler PO, et al .Internal Medicine. Stein JH editor; 1998.

9. Executive Summary of The Third Report of The National Cholesterol Education Program (NCEP) Expert Panel on Detection, Evaluation, And Treatment of High Blood Cholesterol In Adults (Adult Treatment Panel III). Jama. 2001;285(19):2486-97.

10. Dunbar CC, Saul BI, Kassotis J, Badillo L. Usefulness of P-wave morphology during submaximal treadmill exercise to predict coronary artery disease. Am J Cardiol. 2005;96(6):781-3.

11. American Thoracic Society, American College of Chest Physicians . ATS/ACCP Statement on cardiopulmonary exercise testing. Am JRespir Crit Care Med. 2003;167(2):211-77.

12. Lepretre PM, Foster C, Koralsztein JP, Billat VL. Heart rate deflection point as a strategy to defend stroke volume during incremental exercise. J appl Physiol. 2005;98(5):1660-5.

13. Lauer MS. Exercise electrocardiogram testing and prognosis. Novel markers and predictive instruments. Cardiol Clin. 2001;19(3):401-14.

14. Chung EK, Tighe DA. Stress Testing.USA: Blackwell Science; 1997.

15. Pescatello LS, Franklin BA, Fagard R. American College of Sports Medicine position stand. Exercise and hypertension. MedSci Sports Exerc. 2004;36(3):533-53.

16. Amon KW, Richards KL, Crawford MH. Usefulness of the postexercise response of systolic blood pressure in the diagnosis of coronary artery disease. Circulation. 1984;70(6):951-6.

17. McHam SA, Marwick TH, Pashkow FJ, Lauer MS. Delayed systolic blood pressure recovery after graded exercise: an independent correlate of angiographic coronary disease. J Am Coll Cardiol. 1999;34(3):754-9.

18. Abidov A, Rozanski A, Hachamovitch R, Hayes SW, Aboul-Enein F, Cohen I, et al. Prognostic significance of dyspnea in patients referred for cardiac stress testing. N Engl JMed. 2005;353(18):1889-98.

19. Berne RM, Levy MN. Physiology. 4th edSydney: Mosby; 1998.

20. Laragh JH, Brenner BM. Hypertension: Pathophysiology, Diagnosis and Management.New York: Reven Press; 1995.

21. Khan JK, Zola B, Juni JE. Decreased exercise heart rate and blood pressure response in diabetic subjects with cardiac autonomic neuropathy. Diabetes Care. 1986;9(4):389-94.

22. Bernardi L. Clinical evaluation of arterial baroreflex activity in diabetes. Diabet Nutr Metabol Clin Experiment. 2000;13(6):331-40.

23. Stewart KJ, Sung J, Silber HA, et al . Exaggerated Exercise Blood Pressure is related to impaired endothelial vasodilator function. Am J Hypertens. 2004;17(4):314-20.

24. Einhorn D, Reaven GM, Cobin RH, Ford E, Ganda OP, Handelsman Y, et al. American College of Endocrinology position statement on the insulin resistance syndrome. Endocr Pract. 2003;9(3):237-52.

25. Poirier P, Garneau C, Bogaty P. Impact of left ventricular diastolic dysfunction on maximal treadmill performance in normotensive subjects with well controlled type 2 diabetes mellitus. Am J Cardiol. 2000;85(4):473-7.
26. Bell DSH. Diabetic Cardiomyopathy. Diabetes Care. 2003;26(10):2949-50.

27. Bacon SL, Lavoie KL, Campbell TS, Fleet R, Arsenault A, Ditto B. The role of ischaemia and pain in the blood pressure response to exercise stress testing in patients with coronary heart disease. J Hum Hypertens. 2006.

28. Yerkey MW, Kernis SJ, Franklin BA. Renal dysfunction and acceleration of coronary disease. Heart. 2004;90(8):961-6.

29. Mueller C, Neumann FJ, RPerruchoud AP. Renal function and long-term mortality after unstable angina/non-STsegment elevation myocardial infarction treated very early and predominantly with percutaneous coronary intervention. Heart. 2004;90(8):902-7.

30. Mircoli L, Rivera R, Giuseppe B. Influence of left ventricular mass, uremia and hypertension on vagal tachycardic reserve. J Hypertens. 2003;21(8):1547-53.

31. Thambyrajah J, Landray MJ, McGlynn FJ. abnormalities in endothelial function in patients with predialysis renal failure. Heart. 2000;83(2):205-9.

32. Regensteiner JC, Sipple J, McFarling ET. Effects of non-insulin dependent diabetes mellitus on exercise performance. Med Sci Sports Exerc. 1995;27(2):875-81.

33. Masuda I, Sato I. Exercise testing clinical and angiographic characteristics of anginal patients with and without diabetes mellitus. Angiology. 1989;40(7):656-65.

34. Disturbed Hemodynamic Cardiac Exercise Stress Test Reponse in non-smoking, Normolipidemic, Normotensive Diabetic Subjects. In: Khoshdel AR, Carney SI, White S editors. Cardiovascular disease in the 21st century: Shaping the future. 2006 Sydney.

35. Lauer MS, Francis GS, Okin PM, Pashkow FJ, Snader CE, Marwick TH. Impaired chronotropic response to exercise stress testing as a predictor of mortality. JAMA. 1999;281(6):524-9.

36. Williams SG, Jackson M, Ng LL, Barker D, Patwala A, Tan LB. Exercise duration and peak systolic blood pressure are predictive of mortality in ambulatory patients with mild-moderate chronic heart failure. Cardiology. 2005;104(4):221-6.

37. Fletcher GF, Balady G, Froelicher VF, Hartley LH, Haskell WL, Pollock ML. Exercise standards. A statement for healthcare professionals from the American Heart Association. Writing Group. Circulation. 1995;91(2):580-615.

38. Gobel FL, Norstrom LA, Nelson RR, Jorgensen CR, Wang Y. The rate-pressure product as an index of myocardial oxygen consumption during exercise in patients with angina pectoris. Circulation. 1978;57(3):549-56.

39. Brantsma AH, Bakker SJ, Hillege HL, de Zeeuw D, de Jong PE, Gansevoort RT. Urinary albumin excretion and its relation with Creactive protein and the metabolic syndrome in the prediction of type 2 diabetes. Diabetes Care. 2005;28(10):2525-30.

40. Estacio RO, Regensteiner JG, Wolfel EE. The association between diabetic complications and exercise capacity in NIDDM patients. Diabetes Care. 1998;21(2):291-5.

41. Kelbaek H, Jensen T. Impaired left ventricular function in IDDM patients with increased urinary albumin excretion. Scand J Clin Lab Invest. 1991;51:467-73.

42. Sampson MJ, Chambers JB. Abnormal diastolic function in patients with type 1 diabetes and early nephropathy. British Heart Journal.1990;64:266-71.

43. Rutter MK, Wahid ST, McComb JM, Marshall SM. Significance of silent ischemia and microalbuminuria in predicting coronary events in asymptomatic patients with type 2 diabetes. J Am Coll Cardiol. 2002;40(1):56-61.

44. Rutter MK, McComb JM, Brady S, Marshall SM. Silent myocardial ischemia and microalbuminuria in asymptomatic subjects with non-insulin-dependent diabetes mellitus. Am J Cardiol. 1999;83(1):27-31.

45. Al-Attar AT, Mahussain SA, Sadanandan S. Cardiac tests in asymptomatic type 2 diabetics. Med Princ Pract. 2002;11(4):171-5.

46. Ala-Houhala I. Effects of exercise on glomerular passage of macromolecules in patients with diabetic nephropathy and in healthy subjects. Scand J Clin Lab Invest. 1990;50(1):27-33.

47. Hidaka S, Kaneko O, Shirai M, Kojima K, Igarashi Y, Oda K, et al. Do obesity and non-insulin dependent diabetes mellitus ag- 
Khoshdel AR et al.

gravate exercise-induced microproteinuria? Clin Chim Acta. 1998;275(2):115-26.

48. Agarwal RP, Thanvi I, Vachhani G, Kochar DK, Rastogi A. Exercise induced proteinuria as an early indicator of diabetic nephropathy. JAssoc Physicians India. 1998;46(9):772-4.

49. Romanelli G, Giustina A, Cimino A, Valentini U, Agabiti-Rosei E, Muiesan G, et al. Short term effect of captopril on microalbuminuria induced by exercise in normotensive diabetics. Bmj. 1989;298(6669):284-8.

50. Christensen CK. Abnormal albuminuria and blood pressure rise in incipient diabetic nephropathy induced by exercise. Kidney Int. 1984;25(5):819-23.

51. Poulsen PL, Ebbehoj E, Mogensen CE. Lisinopril reduces albuminuria during exercise in low grade microalbuminuric type 1 diabetic patients: a double blind randomized study.J Intern Med. 2001;249(5):433-40.

52. Khoshdel AR, Carney SL, Nair BR, Gillies A. Better management of cardiovascular diseases by pulse wave velocity: combining clinical practice with clinical research using evidence-based medicine. Clin Med Res. 2007;5(1):45-52.

53. Khoshdel AR, Thakkinstian A, Carney SL, Attia J. Estimation of an age-specific reference interval for pulse wave velocity: a metaanalysis. JHypertens. 2006;24(7):1231-7.

54. Khoshdel AR, Carney SL. Arterial stiffness in kidney transplant recipients: an overview of methodology and applications. Urol J. 2008;5(1):3-14.

55. Amar J, Ruidavets JB, Chamontin B, Drouet L, Ferrieres J. Arterial stiffness and cardiovascular risk factors in a population-based study. J Hypertens. 2001;19(3):381-7.

56. Blacher J, Pannier B, Guerin AP, et al . Carotid Arterial Stiffness as a predictor of cardiovascular and all-cause mortality in endstage renal disease. Hypertension. 1998;32:570-4.

57. Khoshdel AR, Carney SL, Gillies A, Mourad A, Nanra R, Jones B, et al. The impact of diabetes mellitus on arterial stiffness in end-stage renal disease (Abstract). Nephrology. 2005;10(Supplement):A21.

58. Khoshdel AR, Carney SL, White S. Disturbed hemodynamic cardiac exercise stress test response in non-smoking, normolipidemic, normotensive, diabetic subjects. Diabetes Res Clin Pract. 2006.

59. Cantwell JD, Murray PM, Thomas RJ. Current management of severe exercise-related cardiac events. Chest.1988;93(6):1264-9.

60. Rodgers GP, Ayanian JZ, Balady G, Beasley JW, Brown KA, Gervino EV, et al. American College of Cardiology/American Heart Association Clinical Competence Statement on Stress Testing. A Report of the American College of Cardiology/American Heart Association/American College of Physicians-American Society of Internal Medicine Task Force on Clinical Competence. Circulation. 2000;102(14):1726-38.

61. Zafar-ul-Islam, Waris J, Kango ZA. Use of Exercise Tolerance Test and Thallium Stress Test in the Diagnosis of Ischemic Heart Disease in Soldiers.J College Physician Surg Pakistan. 2009;19(7):406-9.

62. Yamgushi M, Shimizo M, Ino H. Diagnostic usefullness of the post-exercise systolic blood pressure response for the detection of coronary artery disease in patients with diabetes mellitus. Japan Circulat J. 2000;64(12):949-52.

63. Anavekar NS, McMurray JJ, Velazquez EJ, Solomon SD, Kober L, Rouleau JL, et al. Relation between renal dysfunction and cardiovascular outcomes after myocardial infarction. $N$ Engl J Med. 2004;351(13):1285-95.

64. Moyer VA. Screening for Coronary Heart Disease With Electrocardiography: U.S. Preventive Services Task Force Recommendation Statement. Ann Intern Med. 2012;157.

65. Bacci S, Villella M, Villella A, Langialonga T, Grilli M, Rauseo A et al. Screening for silent myocardial ischaemia in type 2 diabetic patients with additional atherogenic risk factors: applicability and accuracy of the exercise stress test. Eur J Endocrinol. 2002;147(5):649-54.

66. Faglia E, Favales F, Calia P, Paleari F, Segalini G, Gamba PL, et al Cardiac events in 735 type 2 diabetic patients who underwent screening for unknown asymptomatic coronary heart disease: 5-year follow-up report from the Milan Study on Atherosclerosis and Diabetes (MiSAD). Diabetes Care. 2002;25(11):2032-6.
67. Mark DB, Shaw L, Harrell F E Jr. Prognostic value of a treadmill exercise score in outpatients with suspected coronary artery disease. NEngl J Med.1991;325(12):849-53.

68. Jouven X, Empana JP, Schwartz PJ, Desnos M, Courbon D, Ducimetiere P. Heart-rate profile during exercise as a predictor of sudden death. N Engl J Med. 2005;352(19):1951-8.

69. Lauer MS, Okin PM, Larson MG, Evans JC, Levy D. Impaired heart rate response to graded exercise. Prognostic implications of chronotropic incompetence in the Framingham Heart Study. Circulation. 1996;93(8):1520-6.

70. Hemodynamic Response to Exercise Predicts the Development of Severe Renal Failure. In: Khoshdel AR, Carney SL editors. . 14th World Congress on Heart Disease. 2008 Toronto, CANADA, July 2008. American Heart Association: p. 11.

71. Hatzfeld JJ, La Veist TA, Gastone-Johnsson FG. Racial/Ethnic Disparities in the Prevalence of Selected Chronic Diseases Among US Air Force Members, 2008. Prev Chronic Dis. 2012;9:110136.

72. Paris RM, Bedno SA, Krauss MR, et al. Weighing in on Type 2 Diabetes in the Military. Diabetes Care. 2001;24:1894-8.

73. Irvani S, Sabayan B, Sedaghat S, Khoshdel AR, et al. . The Association of Elevated Serum Alanine Aminotransferase with Metabolic Syndrome in A Military Population in Southern Iran. Iran Cardiovasc Res J. 2010;4(2):74-9.

74. Khoshdel AR, Seyed Jafari SM, Heydari ST, et al. The Prevalence of Cardiovascular Disease Risk Factors, and Metabolic Syndrome among Iranian Military Parachutists. Int Cardiovasc Res J. 2012;6(2):51-5.

75. Kabir MA, Hossain MR, Hague MF, et al. Prevalence of metabolic syndrome in Army personnel Residing in Bogra Cantonment. JAFMC Bagladesh. 2012;8(2):47-51.

76. Heydari ST, Khoshdel AR, Sabayan B, Abtahi B. Prevalence of Cardiovascular Risk Factors Among Military Personnel in Southern Iran. Iran Cardiovasc Res J. 2010;4(1):22-7.

77. Varma PP, Raman DK, Ramakrishnan TS, et al. Prevalence of Early Stages of Chronic Kidney Disease in Healthy Army Personnel. MJAFI. 2011;67:9-14.

78. Knoflach M, KiechlS, Kind M. Cardiovascular Risk Factors and Atherosclerosis in Young Males: ARMY . Circulation. 2003;108:1064-9.

79. Grundy SM, Howard B, Smith S Jr., Eckel R, Redberg R, Bonow RO. Prevention Conference VI: Diabetes and Cardiovascular Disease: executive summary: conference proceeding for healthcare professionals from a special writing group of the American Heart Association. Circulation. 2002;105(18):2231-9.

80. Knapp RG, Miller MC. Clinical Epidemiology and Biostatistics.Baltimore, Maryland: William \& Wilkins; 1992.

81. Gazzaruso C, Giordanetti S, De Amici E, Bertone G, Falcone C, Geroldi D, et al. Relationship between erectile dysfunction and silent myocardial ischemia in apparently uncomplicated type 2 diabetic patients. Circulation. 2004;110(1):22-6.

82. Casiglia E, Zanette G, Mazza A, Donadon V, Donada C, Pizziol A, et al. Cardiovascular mortality in non-insulin-dependent diabetes mellitus. A controlled study among 683 diabetics and 683 age- and sex-matched normal subjects. Eur J Epidemiol. 2000;16(7):677-84.

83. Tuttle KR, Puhlman ME, Cooney SK, Short R. Urinary albumin and insulin as predictors of coronary artery disease: An angiographic study. Am J Kidney Dis. 1999;34(5):918-25.

84. Raxwal V, Shetler K, Morise A, Do D, Myers J, Atwood JE, et al. Simple treadmill score to diagnose coronary disease. Chest. 2001;119(6):1933-40.

85. The sixth report of the Joint National Committee on prevention, detection, evaluation, and treatment of high blood pressure Arch Intern Med. 1997;157(21):2413-46.

86. Richardson MT, Holly RG, Amsterdam EA, Wang MQ. The value of ten common exercise tolerance test measures in predicting coronary disease in symptomatic females. Cardiology. 1995;86(3):243-8.

87. Eshtiaghi R, Khoshdel A. Serum Melatonin Level Disturbance is Related to Metabolic Syndrome and Subclinical Arterial Dysfunction in Shift Working Healthy Men. Metabol Syndrome Relat Dis. 2013;2(2):100128.

88. Greenland P, Gaziano JM. Clinical practice. Selecting asymptomatic patients for coronary computed tomography or electrocar- 


\section{Khoshdel AR et al.}

diographic exercise testing. N Engl J Med. 2003;349(5):465-73.

89. Zoccali C. Cardiorenal risk as a new frontier of nephrology: research needs and areas for intervention. Nephrol Dial Transplant. 2002;1(Suppl 11):50-4.
90. Silverberg DS, Wexler D, Blum M. The effect of correction of anemia in diabetics and non-diabetics with severe resistant congestive heart failure by subcutaneous erythropoietin and intravenous iron. Nephrol Dial Transplant. 2003;18(1):141-6. 